\section{REVIEWER'S COMMENTS}

The manuscript by Resnick, Wang, and Kaplan is a very interesting example of how a "cognitive" skill can be analyzed in behavioral terms. The authors have laid out in a very reasonable fashion the steps, components, and objectives of an introductory mathematics curriculum. It is a remarkable example of finegrain analysis of an abstract and important concept (numerosity), in very worthwhile and comprehensive levels of its operation (addition, subtraction, equations, etc.). The analysis of a "concept" such as that (or those) into objective behaviors is a worthwhile example for quite a lot of our audience. The probably clever sequencing within this analysis also adds to its educational value, as an example of good programming per se. The relevance to classroom procedure is very good for a large part of our audience.

Some discussion is included describing how the data that are accumulated from the continuing testing program could be used as a dependent variable for classroom research dealing with both curricular and non-curricular variables. One of the most difficult problems of classroom research today is the lack of a standard dependent variable. Standardized tests that sample skills only once a year are clearly insensitive and inadequate. The behavioral analysis provided by Resnick, Wang, and Kaplan, together with Wang's materials, could very well be a large step toward a sensitive and comprehensive continuous measure of skill acquisition in mathematical behavior.

On the negative side, it presents no experimental data, and requires many pages. It may invite a flood of similar articles, almost certainly of lesser quality, which $J A B A$ would be disposed not to accept. However, this one Invited Article makes an example of considerable value, which may prompt experimental analyses of academic curricula. 